\title{
Writing research protocols: An innovative approach
}

\author{
A Sheikh and ML Levy on behalf of the General Practitioners In Asthma Group
}

\begin{abstract}
Aims: To develop a mechanism to write three research protocols within 10 hours while simultaneously enhancing the research protocol writing skills of participants.
\end{abstract}

Method: Members of the General Practitioners In Asthma Group (GPIAG) were invited to attend a research protocol workshop with pre- and postworkshop questionnaires. The main outcome measures were the development of three detailed research protocols and improvement in participants' perceived research protocol writing skills.

Results: Three detailed research protocols were completed within the time allocated. Thirty $(88 \%)$ participants completed pre- and post-workshop questionnaires. Participants believed there were improvements in their ability to formulate an answerable research question $(p<0.01)$, choose an appropriate methodology to answer the question $(p<0.01)$, choose appropriate outcome measures $(p=0.03)$, choose appropriate statistical methods $(p=0.01)$, devise a research timetable $(p<0.01)$, and improve their overall ability to write a research protocol $(p<0.01)$.

Conclusion: It is possible to identify a mechanism, based on a weekend workshop, to write accelerated research protocols whilst simultaneously significantly increasing the research protocol writing skills of participants. This approach has potential to promote the development of research skills within primary care.

\section{INTRODUCTION}

Although over $90 \%$ of all doctor-patient interactions take place within general practice, only a very small proportion of medical research is planned or undertaken in primary care. ${ }^{1}$ In order to promote and develop the discipline of evidencebased medicine, there is a pressing need to increase the research capacity of primary care ${ }^{2,3}$ while simultaneously recognising the time constraints faced by general practitioners (GPs) and their teams. Important developments thus far include: the formation of academic departments of general practice, the establishment of a number of GP research networks, funding of GP academic fellowships, and the availability of money specifically earmarked for primary care research. 4,5 The GPIAG is a special interest professional group committed to improving patient care and promoting high quality research in the field of respiratory medicine. ${ }^{6}$ It has been in existence since 1987 , currently has a membership of 780, and has recently established The GPIAG Chair in Primary Care Respiratory Medicine at Aberdeen University.

Developing and writing a study protocol is a fundamental step in the research process, requiring considerable time and expertise. ${ }^{7.8}$ We report an initiative in which the GPIAG sought to develop and implement a mechanism to write three research protocols, within a weekend workshop, while simultaneously improving the perceived study protocol writing skills of those participating.

\section{METHODS}

All 780 members of the GPIAG were sent written invitations to participate in the Research Protocol Workshop, which was held over a weekend in London in December 1998.

\section{Pre-workshop preparation}

Ideas for research, of relevance to respiratory medicine and primary care, were sought from all those who declared an intention to attend under the following headings:

1. The nature of the problem and the possible research area

2. The idea, refined as far as possible, into a research question

3. Why this area of research is important to primary care

4. In which way will the answer to this question/problem change the way we work in primary care, or why the answer would benefit our patients

5. Ways in which the problem may be approached

6. The possibility of obtaining funding for this particular idea.

Submissions of these outline research proposals were sought on the understanding that the 'ownership' of a study resulting from an idea would be transferred to the GPIAG. We undertook to offer a lead role to those individuals whose ideas were selected to be developed at the workshop.

Seven GPIAG members, a research facilitator and a specialist respiratory nurse with research experience, were identified as 'workshop group leaders'. All research proposals were circulated electronically to the nine group leaders. Three group leaders were allocated to each of the three protocol working groups. The group leaders were invited to choose two research ideas to be presented to each of their protocol working groups.

Two weeks before the workshop all delegates (group leaders and participants) were asked to complete a structured questionnaire enquiring about particular areas of research expertise. Declared skills (previous research expertise, peer reviewed published papers, literature search skills, and critical reading ability) were used to divide participants into three groups to ensure a broad range of research skills in each.

\section{Resources for the workshop}

A medical statistician was present on the first day to offer assistance to the groups. Each of the three groups were provided with a stand-alone personal computer, a printer and modem facilities to access
Aziz Sheikh

Clinical Research Fellow

Department of Primary Health Care and General Practice, Imperial College School of Medicine, Norfolk Place, London, W2 1PG, UK

Mark Levy

General Practitioner

The Kenton Bridge Medical Centre, 155-175 Kenton Road, Kenton, Middlesex HA3 0YX, UK

Correspondence to:

Dr ML Levy marklevy@gpiagasthma.org

Date received: 25/06/99 Date accepted: 30/06/99

Asthma Gen Pract 1999;7(3):39-42 
MEDLINE and EMBASE databases (by arrangement with the librarian of the British Medical Association) as well as the World Wide Web.

\section{Mechanism of running the workshop}

After a brief introduction to the aims of the weekend, participants dispersed into their three, pre-allocated, protocol-working groups (the rationale behind group allocation was clearly explained to all participants). The group leaders presented the two research questions to their respective groups; groups had to choose one question and develop this into a research protocol within the allocated 10 hours of structured time for group work.

\section{Evaluation and outcomes}

The primary outcome measure was the production of three completed research protocols, to include a research question (hypothesis), detailed study design, selection criteria, outcome measures, statistical methods (incorporating sample size calculations and data analysis), costing of study, and a project timetable. Additionally, each group was expected to identify a research team with a view to applying for funding and seeing the project through to completion.

The secondary outcome was to evaluate changes in the self-rated research skills of participants. All participants (including group leaders) were asked to

Table 1 Number of previous peer-reviewed publications by participants $(n=30)$

$\begin{array}{lrr}\text { Number of publications } & \text { Frequency } \\ 0 & 14 & (47 \%) \\ 1-5 & 8 & (30 \%) \\ 6-10 & 1 & (3 \%) \\ 11-20 & 3 & (10 \%) \\ >20 & 3 & (10 \%)\end{array}$

Table 2

Most commonly cited reasons for attending the workshop

Increase knowledge of research methodology

Meeting colleagues and 'networking'

To develop a research protocol

Increase computing and information technology skills

Practical involvement with research complete a semi-

structured questionnaire, immediately before and after the workshop, documenting their perceived protocol writing skills on a six point Likert scale. Questionnaires were anonymous but numbered in pairs to allow comparisons between pre and postworkshop responses. The pre-questionnaires were completed and handed in before the first workshop session. Paired responses were compared using the Wilcoxon matched-pair sign-rank test.

Summary statistics have been presented as

\section{Table 3 Most commonly cited benefits of attending the workshop}

mean scores, rather than medians, as they convey more sensitively the change in delegates' responses. SPSS for Windows version 7.5 was used for all statistical analysis.

\section{RESULTS}

Of the 66 GPIAG members that originally declared an interest to attend, 34 registered on the first day, of whom 31 stayed for the entire workshop. Preand post-workshop questionnaires were completed by $88.2 \%(30 / 34)$ of participants.

\section{Research questions}

In total 10 research questions were submitted to the workshop coordinator. The group leaders for each of the three groups were able to agree on two research questions for presentation to their respective groups.

\section{Research protocols}

All three groups were able successfully to choose a research question and complete a detailed draft study protocol along the required lines. Each group, however, recognised that the protocols needed further refining before they were able to bid for funding. Each of the groups were able to identify a study team with a commitment to take the project forward.

\section{Workshop participants and their research skills} The mean age of delegates was 43.7 years (SD 8.05). Over $70 \%(22 / 30)$ of those attending were male. Sixteen delegates (53.6\%) indicated that they had previously authored or co-authored a research paper that had been published in a peer-reviewed journal. A more detailed breakdown of the number of publications is given in Table 1 .

\section{Reasons for attending}

The five most commonly cited reasons for attending the workshop are listed in Table 2. Other reasons cited included sharing research ideas, improving presentation skills, improving the care of asthmatic patients, improving statistical skills and finding out about the research activities of colleagues.

\section{Benefits of attending}

The five most commonly cited benefits of attending the workshop are listed in Table 3. Other reasons cited included appreciating the difficulties in formulating a research question, improvement in medical knowledge, the importance of having skilled group leaders, enjoyment, improved statistical skills and insight into research culture.

\section{Protocol writing and research skills}

Participants believed that there were improvements in their overall ability to write a research protocol (mean scores $3.20 v s$ 3.90, $p<0.01$ ). A detailed analysis of how participants rated their skills regarding different aspects of protocol writing are detailed in Table 4 . Twenty-eight $(93 \%)$ participants considered themselves to have improved in at least one of the protocol writing categories enquired about.

Participants were also asked about how they rated their overall research skills (Table 5). Similar 
magnitudes of improvements were seen when comparing group leaders with non-group leaders.

Twenty-nine (96.7\%) participants felt that the workshop was well organised, with 25/30 (83\%) stating that they enjoyed the meeting. Over 90\% (28/30) of participants said that they would attend a similar meeting again, and when asked if the GPIAG should organise further protocol workshops $90 \%$ (27/30) gave a positive response. Negative comments were minimal, focusing mainly on the choice of venue.

\section{DISCUSSION}

We have demonstrated that it is possible to organise a structured teaching programme, based on a weekend workshop, that allows detailed research protocols to be rapidly drafted from a pre-identified research question, while simultaneously significantly enhancing the perceived research protocol writing skills of workshop participants. We would have liked to measure actual protocol writing skills objectively, but due to time constraints this was not feasible. However, as many of the participants had some previous experience in writing research protocols and undertaking research, and there was no significant difference in the degree of perceived improvement between those with and without research experience, the perceived skills are likely to be a reasonably accurate reflection of actual skills.

Although we are very pleased with the outcome, there is one major 'pre-workshop' enhancement we would recommend for anyone intending to undertake a similar project; this is to inform participants in advance of the research questions already selected by group leaders. This would enable participants to undertake literature searches, obtain any relevant instruments, and allow them to make a more informed choice regarding the topic chosen for detailed development.

Care has to be taken to make the environment nonthreatening, particularly for those with limited research experience. Based on the participants' positive comments regarding the workshop, and their declared commitment to attend future similar workshops, it appears that this aim was successfully achieved. Promoting a research culture within primary care is a major hurdle that needs to be overcome in order to promote the development and practice of evidence-based general practice. Innovative research methods need to be identified which give primary care workers feelings of ownership of research, provide opportunity for GP input into the design of a proposed research programme and which, at the same time, recognise the time constraints under which most GPs currently work. Although the participants of this workshop were a highly selected group of primary care workers, it appears that the approach we took has the potential to achieve these objectives. The participatory action process described may suit special interest GP groups, theme research groups in many primary care research networks, and the newly emerging primary care groups to facilitate the promotion of better locality-based research.

\section{Sources of funding}

Funded by Astra Pharmaceuticals.

\section{Workshop steering group}

Coordinator and

workshop development: Mark Levy

Workshop Chairman: Dermot Ryan

Group Leaders: Sean Hilton, Gaynor Hoskins, Mark Levy,

Colin McCowan, Robert McKinley, Linda Pearce, David Price, Aziz Sheikh and Mike Thomas

Statistician: Sally Kerry

Secretariat: Medical Marketing Interface 


\section{Acknowledgements}

We are grateful to Adrian Cook, Dr Sangeeta Dhami, and Professors Sean Hilton, Brian Hurwitz and Bonnie Sibbald for commenting on earlier drafts of this paper.

\section{References}

1. Mant D. R\&D in primary care - a NHS priority. $B r J$ Gen Pract 1998;48:871

2. Department of Health. $R \& D$ in primary care. National Working Group Report. London: HMSO, 1997

3. Medical Research Council. Primary health care. Topic review. London: MRC, 1997

4. Department of Health. Primary care: Delivering the future. London: HMSO, 1996

5. Radda G. Primary care research: The MRC's proposals Br J Gen Pract 1998;48:872

6. General Practitioners In Asthma Group. Available from URL: www.gpiag-asthma.org/asthma/GPIAG/ welcome.htm

7. Pocock SJ. Clinical trials: A practical approach. Bath: Wiley, 1983:28-49

8. Carter Y. Writing a research protocol and getting funded. In: Carter Y, Thomas C, eds. Research methods in primary care. Oxford: Radcliffe, 1997:19-30 